\title{
Estudo da relação entre alimentos cariogênicos da merenda escolar e a experiência de cárie em crianças atendidas em uma escola do Recife
}

\author{
Study of the relationship between cariogenic foods in school meals and caries experience in children \\ attended at a school in Recife
}

Estudio de la relación entre los alimentos cariogénicos en las comidas escolares y la experiencia de caries en niños que asisten a una escuela de Recife

\author{
Luiz Carllos Almeida Felix \\ ORCID: https://orcid.org/0000-0002-9156-4623 \\ Universidade Federal de Pernambuco, Brasil \\ E-mail: luiz_karllos@hotmail.com \\ Cintia Carliene Santos de Oliveira \\ ORCID: https://orcid.org/0000-0002-0971-1146 \\ Universidade Federal de Pernambuco, Brasil \\ E-mail: cintia2380@ hotmail.com \\ Lucas Viana Silva Ramos \\ ORCID: https://orcid.org/0000-0002-5818-1575 \\ Universidade Federal de Pernambuco, Brasil \\ E-mail: lucaaschin@gmail.com \\ Luiz Fernando Alves de Lima \\ ORCID: https://orcid.org/0000-0002-5515-3261 \\ Universidade Federal de Pernambuco, Brasil \\ E-mail: luizlimafernando@hotmail.com \\ João Vítor Queiroz Mendes dos Santos \\ ORCID: https://orcid.org/0000-0002-9554-892X \\ Universidade de Pernambuco, Brasil \\ E-mail: jvitorqueiroz@ hotmail.com \\ Elizabeth Arruda Carneiro Ponzi \\ ORCID: https://orcid.org/0000-0002-2657-9343 \\ Universidade Federal de Pernambuco, Brasil \\ E-mail: bethcirurgia@yahoo.com.br
}

\begin{abstract}
Resumo
A cárie dentária é uma doença ecológica de origem multifatorial que tem prevalecido durante séculos e continua gerando grande preocupação mundial, apesar de todo monitoramento e controle. A introdução de alimentos cariogênicos na infância, atrelados a hábitos de higiene bucal deficientes contribuem para o desenvolvimento da cárie implicando na qualidade de vida. Este estudo tem como objetivo avaliar a relação dos alimentos cariogênicos da merenda escolar e a experiência de cárie nas crianças atendidas em uma escola pública do Recife. Trata-se de uma pesquisa transversal e analítica com uma população composta por 179 crianças utilizando formulários de marcadores do índice ceo-d e CPO-D e do consumo alimentar. Resultados demostraram uma média de ingestão de carboidratos de $173,01 \mathrm{~g}$, sendo $135,34 \mathrm{~g}(78,23 \%)$ para alimentos fornecidos pela escola e 37,67g $(21,77 \%)$ para os alimentos trazidos de casa. Os valores médios do ceo-d e CPO-D quanto a faixa etária foram de 1,91 e 0,30 para as crianças de 4 a 7 anos, 2,0 e 0,44 para as crianças de 7 a 9 anos, 0,40 e 1,20 para as crianças de 10 a 11 anos. Observou-se com isso, que os índices ceo-d e CPO-D apresentam valores divergentes conforme faixa etária, ratificando a presença de fatores de risco como frequência do consumo de alimentos ricos em carboidratos, má higiene oral e presença de microbiota cariogênico. Uma dieta balanceada, aliada a hábitos adequados de higiene bucal e consultas periódicas ao dentista, torna-se necessário para prevenção da cárie precoce.
\end{abstract}

Palavras-chave: Cárie dentária; Hábitos alimentares, Higiene bucal.

\begin{abstract}
Dental caries is an ecological disease of multifactorial origin that has prevailed for centuries and continues to generate great worldwide concern despite all monitoring and control. The introduction of cariogenic foods in childhood, coupled with deficient oral hygiene habits, contribute to the development of caries, implying quality of life. This study aims to evaluate the relationship between cariogenic foods in school meals and the experience of caries in children attended at a public school in Recife. This is a cross-sectional and analytical research with a population composed of
\end{abstract}


179 children using markers of the ceo-d and DMFT and food consumption index. Results showed an average carbohydrate intake of $173.01 \mathrm{~g}$, of which $135.34 \mathrm{~g}(78.23 \%)$ for food provided by the school and $37.67 \mathrm{~g}(21.77 \%)$ for food brought from home. The mean values of ceo-d and CPO-D for the age group were 1.91 and 0.30 for children with age between 4 to 7 years, 2.0 and 0.44 for children with age between 7 to 9 years, 0.40 and 1.20 for children with age between 10 to 11 years. It was observed that the ceo-d and CPO-D indices present divergent values according to the age group, confirming the presence of risk factors such as frequency of consumption of carbohydrate-rich foods, poor oral hygiene and presence of microbiota cariogenic. A balanced diet, coupled with adequate habits of oral hygiene and periodic visits to the dentist, is necessary for the prevention of early decay.

Keywords: Dental caries, Eating habits, Oral hygiene.

\section{Resumen}

La caries dental es una enfermedad ecológica de origen multifactorial que ha prevalecido durante siglos y sigue generando una gran preocupación, a pesar de todo seguimiento y control. La introducción de alimentos cariogénicos en la infancia, ligada a malos hábitos de higiene bucal, contribuye al desarrollo de caries que redunda en calidad de vida. Este estudio tiene como objetivo evaluar la relación entre los alimentos cariogénicos en las comidas escolares y la experiencia de la caries en niños que asisten a una escuela pública. Se trata de una investigación transversal y analítica con una población de 179 niños utilizando formas de marcadores del índice ceo-d y CPO-D y consumo de alimentos. Los resultados mostraron una ingesta media de carbohidratos de 173,01 g, siendo 135,34 g (78,23\%) para los alimentos proporcionados por la escuela y 37,67 g (21,77\%) para los alimentos traídos de casa. Los valores medios del ceo-d y CPOD para el grupo de edad fueron 1,91 y 0,30 para niños de 4 a 7 años, 2,0 y 0,44 para niños de 7 a 9 años, 0, 40 y 1,20 para niños de 10 a 11 años. años. Se observó que los índices ceo-d y CPO-D presentan valores diferentes según grupo de edad, lo que confirma la presencia de factores de riesgo como frecuencia de consumo de alimentos ricos en carbohidratos, mala higiene bucal y presencia de microbiota cariogénico. Una dieta equilibrada, combinada con hábitos adecuados de higiene bucal y visitas periódicas al dentista, es necesaria para prevenir la caries.

Palabras clave: Caries dental, Hábitos alimentarios, Higiene bucal.

\section{Introdução}

A promoção de saúde bucal para as crianças é de grande importância, além da motivação dos pais. As doenças orais estão entre os problemas mais comuns e difundidos em todo o mundo. A má saúde bucal pode ter um impacto significativo na qualidade de vida das crianças, o que leva a deterioração da saúde. As crianças com má saúde oral são mais susceptíveis a não frequentares as aulas do que aqueles com boa saúde oral (Garbin, Soares, Dócusse, Garbin \& Arcieri, 2005).

As lesões de cárie têm acompanhado o ser humano desde épocas pré-históricas, época em que a doença foi caracterizada como sendo de baixos impactos populacionais termos quantitativos e gravidade. Concomitante com o processo de civilização pode-se observar um aumento na prevalência da doença como resultado da redução do consumo de carboidratos e fibras complexas e a introdução de uma dieta mais rica em gorduras, açúcares e alimentos refinados. Assim, até hoje, a cárie dentária tem sido considerado um grave problema de saúde pública (Cagnani, Barros, Sousa, Oliveira, Zanin \& Flório, 2014).

A cárie dentária é uma doença infecciosa de natureza multifatorial resultante da interação físico-químico, biológico (saliva e dentes), comportamental (hábitos alimentares, higiene bucal, estilo de vida) e modulação, fatores de saúde (saúde geral, socioeconômico e educacional) (Cagnani, Barros, Sousa, Oliveira, Zanin \& Flório, 2014).

A cárie dental trata-se de uma doença infecciosa que mais acomete a cavidade bucal, podendo atingir todas as faixas etárias, desde bebês a idosos, e que está diretamente relacionada à introdução dos carboidratos refinados na dieta da população, principalmente a sacarose, que é considerada o dissacarídeo mais cariogênico, sendo este o mais presente na dieta familiar em quase todo o mundo (Feijó \& Iwasaki, 2014).

Os alimentos cariogênicos que estimulam o desenvolvimento de cárie dentária são os ricos em carboidratos fermentáveis, também chamados de açúcares livres, como o pão branco, bolos, bolachas e doces, por serem ricos em carboidratos fermentáveis. Além disso, esses alimentos não são bons para a nutrição das crianças e competem com alimentos mais nutritivos. Fatores como o tipo de glicídios presentes, a consistência pegajosa ou aderente do alimento (como balas e doces cristalizados) e o tempo que o alimento permanece na boca exercem grande influência na sua capacidade de provocar cáries (Cagnani, Barros, Sousa, Oliveira, Zanin \& Flório, 2014). 
Através do último levantamento epidemiológico de âmbito nacional na área de saúde bucal realizado através do Ministério da Saúde por meio da Política Nacional de Saúde Bucal (Brasil Sorridente), em 2010, foi possível ter um panorama de como está a condição de saúde bucal da população brasileira. Um dos diagnósticos realizados foi através do índice CPO-D e positivamente foi visto que vem havendo uma redução nos índices de cárie com o passar dos anos, mas nas crianças de 5 anos, mesmo com uma redução de $17 \%$ comparando com a mesma pesquisa em 2003, $80 \%$ destes não foram tratados, retratando a importância de campanhas que trabalhem prevenção nesta faixa etária (Brasil, 2010; Brasil, 2011b).

Por tanto, os cirurgiões-dentistas são de grande importância para prevenção e diagnóstico e dos participantes das Equipes de Saúde da Família, os quais precisam orientar e incentivar os programas de prevenção, procurando meios através de políticas direcionadas com o intuito de diminuir os principais fatores de risco das doenças bucais.

Esta pesquisa ocorreu em conformidade a Resolução 466/2012 do Conselho Nacional de Saúde, onde, a coleta de dados só foi realizada após aprovação do projeto pelo Comitê de Ética em Pesquisa envolvendo Seres Humanos da UFPE, e teve como objetivo avaliar a relação dos alimentos cariogênicos da merenda escolar e a experiência de cárie nas crianças atendidas em uma escola pública do Recife.

O presente trabalho avaliou a relação dos alimentos cariogênicos da merenda escolar e a experiência de cárie nas crianças atendidas em uma escola pública do Recife e as suas necessidades, percentual de carboidratos dos alimentos ofertados na merenda escolar e percentual de carboidratos dos alimentos trazidos do domić́lio da criança; bem como avaliar a experiência de cárie e os índices ceo-d e CPO-D.

\section{Cárie dentária}

A cárie é uma doença que atinge, principalmente, as crianças, mas que pode ser observada em diferentes fases da vida (Júnior Lima, Gonçalves \& Correia, 2015). Trata-se de doença multifatorial que está apresentando tendência mundial de declínio, sobretudo em países desenvolvidos (Brasil, 2012).

A lesão de cárie ela por sua vez, compreende a perda de tecidos calcificados dos dentes, caracterizada pela destruição localizada de tecidos dentários mineralizados, decorrente da fermentação de carboidratos da dieta por microrganismos do biofilme dentário (Jorge, 2012).

Seu primeiro sinal clínico é representado pela mancha branca ativa, tendo outros sintomas mais recorrentes como a sensibilidade e dor dentária, em alguns casos o dente cariado é assintomático, principalmente em um estágio inicial ou quando não afeta zonas próximas à polpa. Dentes com cárie profunda poderão apresentar uma dor excruciante, incapacitar função mastigatória ou até mesmo originar infeções com repercussões sistêmicas (Cruz, Gomes Neto, Lima, Silva, \& Hora, 2020).

O processo cariogênico começa com a produção de ácidos, quando o produto de metabolismo bacteriano ocupa a biofilme. A desmineralização da superfície continua até a ação de tamponamento salivar ser capaz de elevar o pH acima do nível cítrico. Posteriormente, a placa dentária se combina com o cálcio e endurece, formando o tártaro ou o cálculo, irritando também a gengiva (Feijó \& Iwasaki, 2014). As propriedades de uma dieta ou de um alimento pode provocar cárie dentária e a cariogenicidade de um alimento varia, dependendo da forma na qual ele é encontrado, da composição de nutrientes, de quando ele é consumido com relação a outros alimentos e líquidos, da duração de sua exposição ao dente e da frequência com que é consumido (Freire, Balbo, Amador \& Sardinha, 2012; Feijó \& Iwasaki, 2014).

Os principais microrganismos causadores da cárie são os Estreptococos do grupo mutans, especialmente o Streptococcus mutans e o Streptococcus sobrinus (Seow, 1998; Parisotto, Stipp, Rodrigues, Mattos-Graner, Costa \& Nobredos-Santos, 2015). Esses patógenos são capazes de colonizar a superfície do dente e produzir ácidos em velocidade superior à capacidade de neutralização do biofilme em ambiente abaixo do pH crítico (menor que 5,5), permitindo a dissolução do esmalte (Biral, Taddei, Passoni \& Palma, 2013). 
Os principais fatores de risco associados ao desenvolvimento de cárie são fatores culturais e socioeconômicos, falta de acesso ao flúor, deficiente controle mecânico do biofilme (placa bacteriana), consumo excessivo e frequente de açúcar e xerostomia (Brasil, 2010).

A influência da dieta na saúde oral, especialmente em relação à cárie, se dá por diversas formas. Problemas na estrutura dos dentes durante a formação das placas dentárias, atrasos na erupção da dentição decídua e alterações nas glândulas salivares podem ser causadas por deficiências nutricionais. Em relação aos efeitos locais diretos, a presença de açúcar na dieta pode causar cáries e a presença de ácidos erosão dentária. A qualidade da dieta tem importante relação com a saúde oral, sendo os tecidos periodontais também beneficiados com uma nutrição adequada (Freire, Balbo, Amador \& Sardinha, 2012). Além disso, a dor causada pela cárie interfere no ato de comer e faz com que as crianças apresentem crescimento mais lento, mais baixo peso e mais distúrbios no sono (Nunes \& Perosa, 2017).

O controle de fatores relacionados com o desenvolvimento e progressão da doença cárie, como consumo de sacarose, presença de microrganismos específicos, condição socioeconômica, acesso a cuidados odontológicos e hábitos dos indivíduos é de fundamental importância para se obter uma redução significativa nos níveis de CPO-D (Brasil, 2010; Feijó \& Iwasaki, 2014).

\section{Práticas alimentares}

A composição dos alimentos e os hábitos dietéticos podem afetar ou não a atividade de cárie. Os alimentos servem como substrato para a fermentação pela atividade da microbiota da placa e consequente formação de ácidos orgânicos capazes de desmineralizar a superfície do dente (Garbin, Chiba, Garbin \& Arcieri, 2011; Jorge, 2012; Freire, Balbo, Amador \& Sardinha, 2012).

A alimentação é um tema bastante discutido quando relacionada a uma vida saudável, em que uma boa dieta associada a ingestão de alimentos saudáveis, boa higienização bucal, correta escovação e uso do fio dental contribuem para prevenção e redução de doenças sistêmicas e manutenção da saúde bucal. As práticas alimentares reconhecidamente cariogênicas são principalmente o consumo de açúcares simples e sua frequência (Júnior Lima, Gonçalves \& Correia, 2015).

As práticas alimentares, especialmente o consumo de carboidratos fermentáveis, possuem destaque na etiologia da cárie dentária. Os carboidratos são parcialmente digeridos na cavidade oral sob a ação da amilase salivar resultando em pequenas moléculas de carboidratos que são facilmente utilizados como substrato energético pelas bactérias da microbiota oral. A fermentação destas moléculas de carboidratos produz ácidos que são responsáveis pela desmineralização do esmalte dentário, e consequente desenvolvimento da cárie (Çolak, Dülgergil, Dalli \& Hamidi, 2013).

Alimentos aos quais são adicionados açúcares, durante o processamento alimentar para alterar o sabor e textura, e alimentos como sumos de fruta, o mel, os xaropes e o próprio açúcar branco ou amarelo têm açúcares livres (mono e dissacarídeos) e por isso são cariogênicos. Alimentos como leite, grãos, vegetais e fruta não estão relacionados com o desenvolvimento da cárie dentária, como evidenciam alguns estudos, sendo por isso considerados alimentos não cariogênicos (Hashim, Williams \& Thomson, 2009; Çolak, Dülgergil, Dalli \& Hamidi, 2013; Cagnani, Barros, Sousa, Oliveira, Zanin \& Flório, 2014).

Comparando a consistência de dietas resistentes e macias demonstraram que os alimentos com baixo potencial cariogênico são aqueles que são os resistentes, ou seja, que estimulam a atividade mastigatória e, consequentemente, a produção de saliva (Hashim, Williams \& Thomson, 2009; Vega, Botiel, Mazo, Torres \& Domínguez, 2014). Frutas e hortaliças cruas, pelo seu alto teor de fibras, são exemplos de alimentos que estimulam o fluxo salivar pelo processo da mastigação (Moreira, 2016). Ainda segundo os autores, alimentos ricos em carboidratos, consistentes e pegajosos como biscoitos recheados, salgadinhos, bolos, são alimentos com alto potencial cariogênico e se adaptam as fissuras e sulcos da estrutura 
dentária.

Os carboidratos abrangem um dos grandes grupos de biomoléculas na natureza além de serem a mais abundante fonte de energia. Eles podem ser chamados, de uma maneira geral, de glicídios, amido ou açúcar e são classificados em monossacarídeos, oligossacarídeos e polissacarídeos (Jorge, 2012).

A frequência de ingestão, o conteúdo de açúcar e a consistência das refeições são, juntos com a higiene bucal, fatores decisivos para a formação de cárie. Em consequência da incompleta maturação pós-eruptiva do esmalte, da higiene bucal deficiente e de condições de alimentação desfavoráveis, os dentes recém erupcionados são mais susceptíveis ao desenvolvimento de cárie. As bebidas açucaradas e/ou ácidas que mesmo depois do primeiro ano de vida são ingeridas em grande quantidade com o uso da mamadeira, de maneira prolongada e frequente, principalmente à noite, geram a "síndrome da mamadeira", um processo de destruição cariosa dos dentes (Garbin, Soares, Dócusse, Garbin \& Arcieri, 2005; Garbin, Chiba, Garbin \& Arcieri, 2011). O medicamentos líquidos de uso pediátrico apresentam teores elevados de sacarose bem como baixo $\mathrm{pH}$ endógeno, apresentando alta cariogenicidade devido a promoção da queda do $\mathrm{pH}$ bucal, permanecendo baixo por períodos maiores de tempo, contribuindo para a desmineralização ou inibindo o processo de desmineralização e remineralização dos dentes (Babu, Doddamani, Naik \& Jagadeesh, 2014).

Uma dieta equilibrada capaz de proporcionar um estado nutricional adequado, certamente contribui para uma desejável saúde oral do indivíduo. Portanto, algumas restrições ou mesmo o excesso de ingestão de dietéticos, podem influenciar os processos de odontogênese (formação dentária), erupção e desenvolvimento da doença de cárie (Batista, Moreira \& Corso, 2007). Para Feijó (2014), os programas de prevenção de cáries se concentram em uma dieta balanceada, modificação das fontes e quantidades de carboidratos fermentáveis e integração de práticas de higiene oral no estilo de vida das pessoas.

\section{Metodologia}

O projeto desta pesquisa foi submetido ao Comitê de Ética em Pesquisa em Seres Humanos do Centro de Ciências da Saúde/UFPE, através do Certificado de Apresentação para Apreciação Ética (CAAE) nº: 65852017.9.0000.5208, em 17/01/2017, sendo aprovado em 06/04/2017, com o parecer de número 2.004.251.

A pesquisa tratou-se de um estudo epidemiológico observacional analítico do tipo transversal.

\section{População/amostra/ período de estudo}

A população do estudo foi composta por 179 estudantes de 4 a 11 anos matriculados da educação infantil ao $4^{\circ}$ ano em uma escola da rede pública de ensino do Recife-PE, de ambos os gêneros, com um número de 71 estudantes excluídos. A amostragem foi intencional e por conveniência no período fevereiro a maio de 2018. O Cálculo estimado prévio para análise foi de 250 crianças.

\section{Variáveis}

Variável dependente ou desfecho:

- Consumo de alimentos cariogênicos;

- índice de cárie

\section{Variáveis independentes}

- Gênero; Idade (em anos); Escolaridade dos pais; Idades dos pais; Chefe da família; Alimentos consumidos na escola e os alimentos trazidos de casa. 


\section{Critérios de elegibilidade/ critério de inclusão/ critério de exclusão}

Crianças de 4 a 11 anos regularmente matriculadas na escola. Foram incluídas no estudo as crianças de ambos os gêneros, cujos pais aceitaram que seus filhos participassem do estudo, assinando o Termo de Consentimento Livre e Esclarecido - TCLE (para responsável legal pelo menor de 18 anos - resolução 466/12).

\section{Instrumentos para a coleta de dados}

Os instrumentos para coleta de dados foram divididos em três módulos, em que os questionários foram enviados aos pais juntamente com o TCLE por meio de agenda escolar.

O $1^{\circ}$ questionário enviado aos pais no período de março/abril abordou os aspectos sociodemográficos como informações pessoais da criança e dos pais e dos hábitos alimentares na escola, onde na impossibilidade do responsável pela criança responder as questões os pesquisadores (responsáveis pela pesquisa) realizaram as abordagens no momento em que estes foram buscar as crianças na escola. $\mathrm{O} 2^{\circ}$ formulário consistiu em parte do questionário preconizado pelo Ministério da Saúde (fonte: SB Brasil), para levantamento epidemiológico básico de saúde bucal no período de abril/maio, contendo questões de informações gerais da criança. E o $3^{\circ}$ formulário compreendeu em um formulário adaptado para registro dos alimentos consumidos na escola no período de abril/maio, com a respectiva análise centesimal de macronutriente (carboidratos) para efeito de comparação com Tabela Brasileira de Composição de Alimentos (fonte: Tabela Brasileira de Composição dos Alimentos).

\section{Calibração para avaliação clínica}

Antes da coleta dos dados, realizou-se um treinamento teórico acerca dos critérios de diagnóstico. A calibração prática foi realizada na Clínica B do Curso de Odontologia da UFPE, por meio de técnicas de diagnóstico de cárie dentária através de exame clínico em pacientes. Esse diagnóstico baseou-se no formulário proposto pelo Ministério da Saúde. Esse sistema de análise foi adotado por ser o mais indicado para estudos epidemiológicos, uma vez que não há necessidade de exame radiográfico, baseando-se apenas na avaliação clínica.

\section{Procedimentos}

Os procedimentos consistiram em ações educativas voltadas à alimentação saudável e higiene dental. Foram realizadas oficinas com atividades lúdicas no período de abril/maio, utilizando imagens, jogos e macro modelos (boca + escova). Não houve nenhuma intervenção quanto a procedimentos clínicos como remoção de tecido cariado, aplicação tópica de flúor e tratamento restaurador atraumático. Nesta etapa foram realizados o levantamento epidemiológico básico de saúde bucal de cada escolar. Estas atividades ocorreram em dias úteis disponibilizados pela direção da escola, nos turnos da manhã e tarde, durante 5 semanas. Por meio de um formulário adaptado foram registrados os alimentos consumidos na escola verificando a análise centesimal de macronutriente (carboidratos) e posterior comparação com dados da Tabela Brasileira de Composição de Alimentos.

\section{Processamento e análise dos dados}

Os dados foram analisados descritivamente e de forma inferencial. A análise descritiva se deu através de frequências absolutas percentuais para as variáveis categóricas e das estatísticas: média, desvio padrão, mediana e percentis para as variáveis numéricas.

Executou-se a análise inferencial através dos testes estatísticos. Em relação às variáveis numéricas foi utilizado teste de Mann-Whitney na comparação de duas categorias e o teste de Kruskal-Wallis na comparação de três categorias. Para avaliar 
associação entre duas variáveis categóricas foi utilizado o teste Qui-quadrado de Pearson ou teste Exato de Fisher nas situações em que a condição para utilização do teste Qui-quadrado não foi verificada. Para avaliar o grau de associação entre duas variáveis numéricas foi obtido o coeficiente de correlação de Spearman e aplicado um teste t-Student específico para a hipótese de correlação nula.

\section{Resultados}

A Tabela 1 apresenta a distribuição dos dados segundo as variáveis de gênero e faixa etária dos 179 alunos entrevistados. O predomínio do gênero foi masculino, representando $57,0 \%$ dos estudantes; quanto à faixa etária, a predominância ocorreu nas crianças com idade média entre 7 a 9 anos (41,3\%), seguido do grupo etário de 4 a 6 anos $(38,5 \%)$ e o menor percentual $(20,0 \%)$ correspondeu aos pesquisados que tinham entre 10 e 11 anos (Tabela 1 ).

Tabela 1 - Distribuição das amostras quanto ao gênero e faixa etária.

\begin{tabular}{lcc}
\hline Variável & $\mathbf{n}$ & $\mathbf{\%}$ \\
TOTAL & $\mathbf{1 7 9}$ & $\mathbf{1 0 0}$ \\
Gênero & & \\
Masculino & 102 & 57,0 \\
Feminino & 77 & 43,0 \\
Faixa etária (anos) & & \\
4 a 6 & 69 & 38,5 \\
7 a 9 & 74 & 41,3 \\
10 a 11 & 36 & 20,1 \\
\hline
\end{tabular}

A Tabela 2 mostra as estatísticas do consumo de carboidratos dos alimentos de uma refeição fornecidos pela escola e dos alimentos trazidos de casa pelas crianças, por meio de média aritmética dos valores em gramas presentes na Tabela de Composição Alimentar utilizada, onde se verificou que os alimentos fornecidos pela escola $(78,23 \%)$, apresentaram uma média de carboidratos bem mais elevada que a dos alimentos trazidos de casa (21,77\%). Essa diferença correspondeu a 56,46\%.

A variabilidade expressa através do desvio padrão se mostrou muito elevada nos alimentos fornecidos pela escola e no total desde que as referidas medidas foram aproximadas ou mais elevadas do que as médias correspondentes e foi razoavelmente elevada nos alimentos trazidos de casa (desvio padrão mais da metade da média correspondente).

O percentual dos alimentos mais consumidos entre os fornecidos pela escola, que variou de $27,9 \%$ a $55,3 \%$, sendo os mais frequentes: frutas e sopa de carne/frango com legumes e macarrão/arroz $(52,5 \%)$ e os menos frequentes foram bolo de bacia e papa com o mesmo percentual $(27,9 \%)$.

Dos alimentos trazidos de casa os três mais citados foram: biscoito recheado $(65,4 \%)$, refrigerante $(33,0 \%)$ e salgadinho de queijo (29,6\%), e os menos citados foram: amendoim, brigadeiro e pudim, cada um citado uma única vez. 
Tabela 2 - Estatísticas do consumo de carboidratos.

\begin{tabular}{|c|c|c|c|c|c|c|}
\hline Variável & Média & Desvio padrão & Mediana & $\mathbf{P 2 5}$ & P75 & $\begin{array}{c}\text { \% da média } \\
\text { Total }\end{array}$ \\
\hline $\begin{array}{l}\text { Alimentos fornecidos pela } \\
\text { escola }\end{array}$ & 135,34 & 162,88 & 49,50 & 26,00 & 373,58 & 78,23 \\
\hline Alimentos trazido de casa & 37,67 & 28,74 & 34,81 & 20,00 & 53,81 & 21,77 \\
\hline Total & 173,01 & 170,89 & 85,28 & 49,41 & 402,08 & \\
\hline
\end{tabular}

Fonte: Autores.

\section{Resultados do CEO-D, CPO-D e respectivos componentes por gênero}

Os resultados da Tabela 3, para a margem de erro fixada na aplicação dos testes estatísticos ( $\mathrm{p}<0,05$ ), não foram registradas diferenças significativas $(\mathrm{p}<0,05)$ entre os gêneros para cada uma das variáveis relativas ao ceo-d, ao CPO-D e seus componentes.

Tabela 3 - Estatísticas do ceo-d, CPO-D e componentes segundo o gênero.

\section{Gênero}

\begin{tabular}{|c|c|c|c|}
\hline \multirow[t]{2}{*}{ Variável } & Masculino & Feminino & Valor de $p$ \\
\hline & Média \pm DP $($ Mediana $)$ & Média \pm DP (Mediana) & \\
\hline
\end{tabular}

\begin{tabular}{|c|c|c|c|}
\hline \multicolumn{4}{|l|}{ Dentes decíduos } \\
\hline Número de crianças & 81 & 57 & \\
\hline Cariados & $1,65 \pm 2,18(1,00)$ & $1,46 \pm 2,07(1,00)$ & $\mathrm{p}^{(1)}=0,983$ \\
\hline Extraído ou indicado para extração & $0,20 \pm 0,68(0,00)$ & $0,18 \pm 0,50(0,00)$ & $\mathrm{p}^{(1)}=0,764$ \\
\hline Obturados & $0,09 \pm 0,36(0,00)$ & $0,21 \pm 0,84(0,00)$ & $\mathrm{p}^{(1)}=0,755$ \\
\hline ceo-d & $1,94 \pm 2,43(1,00)$ & $1,85 \pm 2,79(1,00)$ & $\mathrm{p}^{(1)}=0,955$ \\
\hline \multicolumn{4}{|l|}{ Dentes permanentes } \\
\hline Número de crianças & 71 & 59 & \\
\hline Cariados & $0,54 \pm 1,01(0,00)$ & $0,59 \pm 1,15(0,00)$ & $\mathrm{p}^{(1)}=0,997$ \\
\hline Obturados & $0,06 \pm 0,23(0,00)$ & $0,05 \pm 0,29(0,00)$ & $\mathrm{p}^{(1)}=0,688$ \\
\hline Perdidos & $0,00 \pm 0,00(0,00)$ & $0,03 \pm 0,26(0,00)$ & $\mathrm{p}^{(1)}=0,454$ \\
\hline CPO-D & $0,60 \pm 1,06(0,00)$ & $0,67 \pm 1,28(0,00)$ & $\mathrm{p}^{(1)}=0,926$ \\
\hline
\end{tabular}

(1) Dados cruzados através do teste Mann-Whitney.

Fonte: Autores. 
Nos resultados da Tabela 4 verificou-se que as únicas diferenças significativas entre as faixas etárias foram registradas para o número de dentes permanentes cariados e para o CPO-D e para as referidas variáveis se ressalta que, as médias foram correspondentemente mais elevadas entre as crianças de 10 a 11 anos e menos elevadas entre as que tinham 4 a 6 anos e através dos testes de comparações múltiplas se comprovam diferenças significativas entre a faixa etária 10 a 11 anos das outras duas faixas etárias.

Tabela 4 - Estatísticas do ceo-d, CPO-D e componentes segundo a faixa etária.

\begin{tabular}{|c|c|c|c|c|}
\hline \multirow{3}{*}{ Variável } & \multicolumn{3}{|c|}{ Faixa etária (anos) } & \multirow{3}{*}{ Valor de $p$} \\
\hline & 3 a 6 & 7 a 9 & 10 a 11 & \\
\hline & $\begin{array}{l}\text { Média } \pm \text { DP } \\
\text { (mediana) }\end{array}$ & $\begin{array}{c}\text { Média } \pm \text { DP } \\
\text { (mediana) }\end{array}$ & $\begin{array}{l}\text { Média } \pm \text { DP } \\
\text { (mediana) }\end{array}$ & \\
\hline \multicolumn{5}{|l|}{ Dentes decíduos } \\
\hline Número de crianças & 69 & 64 & 5 & \\
\hline Cariados & $1,65 \pm 2,44(1,00)$ & $1,58 \pm 1,82(1,00)$ & $0,40 \pm 0,55(0,00)$ & $\mathrm{p}^{(1)}=0,407$ \\
\hline $\begin{array}{l}\text { Extraído ou indicado } \\
\text { extração }\end{array}$ & $0,06 \pm 0,29(0,00)$ & $0,34 \pm 0,82(0,00)$ & $0,00 \pm 0,00(0,00)$ & $\mathrm{p}^{(1)}=0,019$ \\
\hline Obturados & $0,20 \pm 0,76(0,00)$ & $0,08 \pm 0,41(0,00)$ & $0,00 \pm 0,00(0,00)$ & $\mathrm{p}^{(1)}=0,526$ \\
\hline ceo-d & $1,91 \pm 2,89(1,00)$ & $2,00 \pm 2,28(1,00)$ & $0,40 \pm 0,55(0,00)$ & $\mathrm{p}^{(1)}=0,347$ \\
\hline \multicolumn{5}{|l|}{ Dentes permanentes } \\
\hline Número de crianças & 20 & 74 & 36 & \\
\hline Cariados & $\begin{array}{c}0,30 \pm 0,73 \\
(0,00)^{(\mathbf{A})}\end{array}$ & $\begin{array}{l}0,36 \pm 0,77 \\
(0,00)^{(\mathbf{A})}\end{array}$ & $\begin{array}{c}1,11 \pm 1,51 \\
(0,00)^{(\mathbf{B})}\end{array}$ & $\mathrm{p}^{(1)}=0,008^{*}$ \\
\hline Obturados & $0,00 \pm 0,00(0,00)$ & $0,08 \pm 0,32(0,00)$ & $0,03 \pm 0,17(0,00)$ & $\mathrm{p}^{(1)}=0,366$ \\
\hline Perdidos & $0,00 \pm 0,00(0,00)$ & $0,00 \pm 0,00(0,00)$ & $0,06 \pm 0,33(0,00)$ & $\mathrm{p}^{(1)}=0,271$ \\
\hline CPO-D & $\begin{array}{c}0,30 \pm 0,73 \\
(0,00)^{(\mathbf{A})}\end{array}$ & $\begin{array}{c}0,44 \pm 0,86 \\
(0,00)^{(\mathbf{A})}\end{array}$ & $\begin{array}{c}1,20 \pm 1,64 \\
(0,50)^{(\mathbf{B})}\end{array}$ & $\mathrm{p}^{(1)}=0,011^{*}$ \\
\hline
\end{tabular}

(*) Diferença significativa a 5\% (1) Através do teste Kruskal Wallis com comparações do referido teste.

Fonte: Os autores.

\section{Presença de cárie segundo variáveis independentes}

A Tabela 5 apresentou os resultados do estudo dos cruzamentos entre a presença ou ausência de cárie (cárie em decíduo ou em permanente) com as variáveis independentes do estudo. Desta tabela não foram verificadas associações significativas $(\mathrm{p}>0,05)$ entre a presença de cárie e as variáveis analisadas. 
Tabela 5 - Presença de cárie segundo as variáveis independentes.

\begin{tabular}{|c|c|c|c|c|c|c|c|c|}
\hline \multirow[t]{3}{*}{ Variável } & \multicolumn{4}{|c|}{ Presença de cárie } & & & & \\
\hline & \multicolumn{2}{|c|}{ Sim } & \multicolumn{2}{|c|}{ Não } & \multicolumn{2}{|c|}{ Grupo total } & Valor de p & \multirow[t]{2}{*}{ OR } \\
\hline & $\mathrm{n}$ & $\%$ & $\mathrm{n}$ & $\%$ & $\mathrm{n}$ & $\%$ & & \\
\hline Total & 100 & $\mathbf{5 5 , 9}$ & 79 & 44,1 & 179 & 100,0 & & \\
\hline Gênero & & & & & & & $\begin{array}{l}\mathrm{p}^{(1)} \quad= \\
0,547\end{array}$ & \\
\hline Masculino & 55 & 53,9 & 47 & 46,1 & 102 & 100,0 & & 1,00 \\
\hline Feminino & & & & & & & & $1,20(0,66 \mathrm{a}$ \\
\hline & 45 & 58,4 & 32 & 41,6 & 77 & 100,0 & & $2,18)$ \\
\hline Faixa etária (anos) & & & & & & & $\begin{array}{l}\mathrm{p}^{(1)}= \\
0,355\end{array}$ & \\
\hline 4 a 6 & & & & & & & & $1,09(0,49 \mathrm{a}$ \\
\hline & 36 & 52,2 & 33 & 47,8 & 69 & 100,0 & & $2,44)$ \\
\hline 7 a 9 & 16 & 627 & 28 & 378 & 74 & 1000 & & $1,64(0,74 \mathrm{a}$ \\
\hline 10 a 11 & 18 & 50,0 & 18 & 50,0 & 36 & 100,0 & & 1,00 \\
\hline $\begin{array}{l}\text { Consumo de } \\
\text { carboidrato }\end{array}$ & & & & & & & & \\
\hline Alimentos da escola & & & & & & & $\begin{array}{l}\mathrm{p}^{(1)}= \\
0,973\end{array}$ & \\
\hline 0 a 26 (P25) & 26 & 55,3 & 21 & 44,7 & 47 & 100,0 & & 1,00 \\
\hline$>26$ a 49,5 (P50) & & & & & & & & $0,92(0,41 \mathrm{a}$ \\
\hline & 24 & 53,3 & 21 & 46,7 & 45 & 100,0 & & $2,10)$ \\
\hline 49,6 a 373,6 (P75) & & & & & & & & $1,06(0,46 \mathrm{a}$ \\
\hline & 25 & 56,8 & 19 & 43,2 & 44 & 100,0 & & $2,43)$ \\
\hline 373,7 ou mais & & & & & & & & $1,12(0,49 \mathrm{a}$ \\
\hline & 25 & 58,1 & 18 & 41,9 & 43 & 100,0 & & $2,59)$ \\
\hline Alimento de casa & & & & & & & $\begin{array}{l}\mathrm{p}^{(1)}= \\
0,970\end{array}$ & \\
\hline 0 a $26(\mathrm{P} 25)$ & 38 & 54,3 & 32 & 45,7 & 70 & 100,0 & & 1,00 \\
\hline$>20$ a $34,8(\mathrm{P} 50)$ & & & & & & & & $1,03(0,38 \mathrm{a}$ \\
\hline & 11 & 55,0 & 9 & 45,0 & 20 & 100,0 & & $2,79)$ \\
\hline 34,9 a $53,8(\mathrm{P} 75)$ & & & & & & & & $1,26(0,59 a$ \\
\hline & 27 & 58,7 & 19 & 41,3 & 46 & 100,0 & & $2,70)$ \\
\hline 53,9 ou mais & & & & & & & & $1,06(0,49 \mathrm{a}$ \\
\hline & 24 & 55,8 & 19 & 44,2 & 43 & 100,0 & & $2,28)$ \\
\hline
\end{tabular}

(1) Através do teste Qui-quadrado de Pearson.

(2) Através do teste Exato de Fisher.

Fonte: Autores.

\section{Perda dentária segundo variáveis independentes}

Verificou-se que a faixa etária, foi a única variável com associação significativa $(\mathrm{p}<0,05)$ entre a presença ou não de perda dentária devida a presença de cárie, e as variáveis analisadas e para esta variável se enfatizou que o percentual com perda dentária foi mais elevado na faixa 7 a 9 anos $(16,2 \%)$ e variou de 2,8\% a 4,3\% nas outras faixas duas faixas etárias. Devido à ocorrência de frequências muito baixas não se apresentou os valores do OR para esta tabela.

\section{Estudo da correlação entre os valores dos consumos de carboidrato e dados do ceo-d e do CPO-D}

Os resultados do coeficiente de correlação entre os dados do ceo-d e do CPO-D com a quantidade de carboidrato dos 
alimentos consumidos na escola, dos alimentos trazidos de casa e com o total de carboidrato consumido onde se verificou que os valores das correlações variaram de $-0,07$ a 0,09 (baixas correlações) e não foram estatisticamente significativamente diferentes de zero $(\mathrm{p}<0,05)$.

\section{Discussão}

A maneira como se conceitua a cárie dentária e seus fatores etiológicos determina a escolha para estabelecer a estratégia preventiva de diagnóstico e tratamento.

A má saúde bucal pode ter um impacto na qualidade de vida das crianças (Garbin, Soares, Dócusse, Garbin \& Arcieri, 2005). Além disso, a cárie é uma doença que atinge, principalmente, as crianças, mas que pode ser observada em diferentes fases da vida. (Júnior Lima, Gonçalves \& Correia, 2015). Os efeitos da cárie podem ser calculados através de dois índices: ceo-d e cpo-d. O índice ceo-d é o que contabiliza o número de elementos dentários decíduos agredido por cáries, elementos retirados e restaurados. Já o índice cpo-d avalia as mesmas variantes em dentes permanentes (Vasconcelos, Gondim, Rodrigues, Lima Neto, \& Valença, 2018).

Pesquisas comprovam que os alimentos servem como substrato para a fermentação pela atividade da microbiota da placa (Çolak, Dülgergil, Dalli \& Hamidi, 2013; Moreira, 2016), entretanto, alguns autores discordam de que não são apenas os alimentos que provocam a desmineralização e remineralização dos dentes, pois os medicamentos líquidos de uso pediátrico apresentam teores elevados de sacarose, demonstrando alta cariogenicidade devido a promoção da queda do pH bucal (Babu, Doddamani, Naik \& Jagadeesh, 2014).

O acometimento de cárie dentária em escolares com dentição decídua e/ou mista, encontrada nesta pesquisa mediante exame clínico foi superior aos resultados encontrados por Gabin et al. (2011), em seu estudo constituído por 75 crianças de 4 a 6 anos de idade, matriculadas em escolas de Araçatuba-SP, bem como foi superior em pesquisa realizadas por Limeira et al. (2010), com 861 crianças de 4 anos de idade, pré-escolares do Recife. Entretanto, valores inferiores foram encontrados para o índice ceo-d quando comparados aos estudos Limeira e colaboradores (2010). Esta pequena diferença na prevalência em relação às demais pesquisas segundo Moreira (2016), varia de acordo com a região, idade, condições socioeconômicas e culturais e depende da interação de fatores como características dos dentes, microbiota e padrão alimentar.

Em relação a avaliação de crianças com dentição mista e/ou permanente, as a experiência de cárie dentária encontrada foi apresentou resultados superiores aos encontrados na avaliação da dentição permanente realizada por Gabin et al (2011), em sua pesquisa com 75 crianças de 4 a 6 anos de idade. Observou-se também, que o número médio de dentes hígidos, em ambas as dentições, foi maior em relação aos componentes dos índices utilizados. Em pesquisa governamental (Brasil, 2012) encontrou-se valores inferiores na variável cariados em um estudo com 197 crianças de faixa etária de 12 anos de idade na cidade do Recife. Para a média CPO-D encontrada na presente pesquisa, a mesma apresentou valores inferiores quando comparado a outras pesquisas (Hashim, Williams \& Thomson, 2009; Brasil, 2012; Vega, Botiel, Mazo, Torres \& Domínguez, 2014; Parisotto, Stipp, Rodrigues, Mattos-Graner, Costa \& Nobre-dos-Santos, 2015). Esse resultado divergente é justificado pela presença de fatores de risco como frequência do consumo de refeições, consumo de alimentos ricos em carboidratos, má higiene, escolaridade dos pais, renda familiar e presença de microbiota cariogênica.

Em relação ao índice ceo-d e CPO-D quanto ao gênero, houve maior experiência de cárie em crianças do gênero feminino e faixa etária de 7 a 9 anos. Já as perdas dentárias as maiores experiências de cárie também corresponderam ao gênero feminino e a faixa etária de 7 a 9 anos. Entretanto, resultados diferentes foram encontrados em pesquisa realizada na cidade do Recife em 267 crianças com idade de 5 anos, onde foram encontradas valores maiores equivalentes a variável cariados e índices ceo-d/CPO-D, porém a variável dentes perdidos apresentou valores inferiores quando comparados a pesquisa corrente (Brasil, 2012). No que concerne ao grupo etário de 12 anos (197 crianças), esses valores correspondentes 
para dentes cariados, perdidos e índices ceo-d/CPO-D foram distintos.

Noutra pesquisa em 2.109 crianças de 5 anos de idade na região Nordeste, a média para dentes cariados correspondeu a 2,55 (88,2\%), 0,08 (2,89\%) para dentes perdidos e a média de ceo-d/CPO-D correspondeu a 2,09\%; no grupo de 12 anos (2.021 crianças), esses valores corresponderam a $(1,81-68,8 \%)$ para dentes cariados, $(0,22-8,4 \%)$ para dentes perdidos e índices ceo-d/CPO-D de (2,63\%) (Brasil, 2012).

Esses valores divergentes, podem ser explicados devido as pesquisas utilizarem variáveis e grupos etários distintos (5 anos e 12 anos apenas na infância), devido ao número das amostras e o que permite uma variação pequenas.

O coeficiente de correlação entre os dados do ceo-d e do CPO-D com a quantidade de carboidrato dos alimentos consumidos na escola, dos alimentos trazidos de casa variaram de 0,07 a 0,09 para ceo-d e de 0,02 a 0,05 para CPO-D, corroborando com estudos realizados por Silva (2007), em crianças de 6 a 8 anos matriculadas em escolas de Matosinhos Portugal, em que foi verificado a relação do aumento do índice CPO-D com a ingestão de alimentos mono e dissacarídeos.

A análise do consumo de alimentos ricos em açúcar demonstrou que o consumo elevado de alimentos como biscoito recheado, refrigerantes, achocolatados, sucos artificiais de frutas, estão associados ao maior índice ceo-d e CPO-D concordando com outra pesquisa (Dresti \& Waes, 2002). Em nosso trabalho correlacionando o consumo de carboidratos com a presença de cárie e perdas dentárias, maiores valores de cárie estão presentes em crianças que consomem os alimentos trazidos de casa $(55,95 \mathrm{~g})$, já alimentos consumidos na escola correspondem a $(55,87)$ corroborando com resultados encontrados por Batista e cols (2007). Nas perdas dentárias por sua vez, os maiores índices encontram-se em crianças que consomem alimentos na escola $(9,1 \mathrm{~g})$, em contrapartida, as crianças que levam os alimentos para a escola apresentaram $(8,88 \mathrm{~g})$. Estudos realizados por Biral et al. (2013) e Hashim e cols. (2009), permitiram detectar que os cardápios das merendas oferecidas nas escolas de Londrina são cariogênicos, o mesmo resultado foi encontrado em nossa pesquisa onde a frequência de ingestão de carboidratos fermentáveis e a ausência de higienização bucal supervisionada após a merenda contribuiu para o aumento dos índices de cárie, concordando com alguns achados (Ardengli, Piovesan \& Antunes, 2013; Vega, Botiel, Mazo, Torres \& Domínguez, 2014), em que o risco de cárie aumenta quando carboidratos fermentáveis são consumidos em alta frequência. Outras investigações (Carlson, 1973; Mahan, 1995) concordaram que os alimentos com baixo potencial cariogênico são aqueles que estimulam a atividade mastigatória, mas os alimentos ricos em carboidratos, consistentes e pegajosos como biscoitos recheados se unem as fissuras e sulcos do dente aumentando o quantitativo de carie, ratificando nosso resultado de $65,4 \%$ de ingestão de biscoito recheado trazido pelos alunos. .

Com relação as condições e necessidade de tratamentos, foi possível observar que a maioria dos dentes presentes estavam sadios (1814 dentes decíduos e 1692 dentes permanentes) não necessitando de tratamento. Entre os dentes decíduos que necessitavam de tratamento, as frequências foram mais elevadas nos dentes: 55, 54, 64, 65, 85, 84, 74 e 75 e nos dentes permanentes foram os primeiros molares $(16,26,36 \mathrm{e} 46)$.

\section{Conclusão}

Os alimentos fornecidos na merenda escolar e os trazidos do domicílio das crianças da instituição apresentou alto índice de carboidratos.

Avaliando o índice cariogênico dos alimentos ofertados na merenda escolar e os alimentos trazidos do domicílio das crianças, estes apresentaram valores cariogênicos elevados, onde se faz necessário maior rigor na seleção e ofertas destes alimentos, atentando para critérios como: potencial cariogênico, valor nutricional, alimentos naturais, visando a prevenção de cárie dentária e outras doenças sistêmicas.

No que tange a relação dos alimentos cariogênicos e a experiência de cárie dentária, foi possível observar grande aumento na frequência das variáveis cárie e perda dentária nos grupos estudados, bem como da prevalência hábitos alimentares 
inadequados, tornando-se evidente a necessidade de maior restrição no consumo desses alimentos ricos em açucares, tendo em vista que tais problemas são passíveis de controle mediante ações educativas de atenção básica.

É interessante para intervenções futuras, além da observação dos fatores dietéticos relacionados a cárie precoce, a associação das mudanças na dieta e qual o impacto dessas modificações nas regressões das lesões cariosas.

\section{Referências}

Ardengli, T. M., Piovesan, C., \& Antunes, J. L. (2013). Desigualdades na prevalência de cárie dentária não tratada em crianças pré-escolares no Brasil. Rev Saúde Pública, 47(3), 129-137.

Babu, K. L., Doddamani, G. M., Naik L. R., \& Jagadeesh, K. N. (2014). Pediatric liquid medicaments - Are they cariogenic? An in vitro study. J Int Soc Prev Community Dent, 4(2), 108-112.

Batista, R. L., Moreira, E. A., \& Corso, A. C. (2007). Food, nutritional status and oral condition of the child. Rev Nutr, 20(2), 191-196.

Biral, A. M., Taddei, J. A., Passoni, D. F., \& Palma, D. (2013). Cárie dentária e práticas alimentares entre crianças de creches do município de São Paulo. Rev Nutr, 26(1), 37-48.

Brasil. (2010). Brasil Sorridente. A saúde bucal levada a sério. Pesquisa Nacional de Saúde Bucal. Ministério da Saúde, Secretaria de Atenção Primária a Saúde, Brasília.

Brasil. (2011). Decreto n. 7.508, de 28 de junho de 2011. Regulamenta a Lei n. 8.080, de 19 de setembro de 1990, para dispor sobre a organização do Sistema Único de Saúde - SUS, o planejamento da saúde, a assistência à saúde e a articulação Inter federativa, e dá outras. Brasil, Diário Oficial da República Federativa do Brasil, Brasília.

Brasil. (2011b). Brasil Sorridente. Principais Resultados. A saúde bucal levada a sério. Pesquisa Nacional de Saúde Bucal. Relatório Final, Ministério da Saúde, Secretaria de Atenção Primária a Saúde, Brasília.

Brasil. (2012). SB Brasil 2010: Pesquisa Nacional de Saúde Bucal: resultados principais. Ministério da Saúde, Secretaria de Atenção Primaria a Saúde, Brasília.

Brasil. (2012). SB BRASIL 2010: Pesquisa Nacional de Saúde Bucal: resultados principais. Editora MS.

Cagnani, A., Barros, A. M., Sousa, L. L., Oliveira, A. M., Zanni, L., \& Florio, F. M. (2014). Association between preference for sweet foods and dental caries. RGO - Rev Gaúcha Odontol, 62(1), 25-29.

Carlson, J. J. (1973). Sugar and production of bacteria in the human mouth. Rev Caries Research, 7(4), $273-282$.

Cruz, A. I., Gomes Neto, M. M., Lima, W. T., Silva, W. A., \& Hora, S. L. (2020). Novos métodos de diagnóstico para detecção da cárie dental - revisão integrativa. Research, Society and Development, 9(10), pp. e7209109160-e7209109178.

Çolak, H., Dulgergil, Ç., Dalli, M., \& Hamidi, M. M. (2013). Early childhood caries update: A review of causes, diagnoses, and treatments. J Nat Sci Biol Med, 4(1), pp. 29-38.

Dresti, D. V., \& Waes, H. V. (2002). Prevenção coletiva, semicoletiva e individual em crianças e adolescentes. In: H. Waes, \& P. Stockli, Odontopediatria (pp. 133-150). Porto Alegre: Artmed.

Feijó, I. S., \& Iwasaki, K. M. (2014). Cárie e dieta alimentar. Rev UNINGÁ Review, 19(3), 44-50.

Freire, M. D., Balbo, P. L., Amador, M. D., \& Sardinha, L. M. (2012). Guias alimentares para a população brasileira: implicações para a Política Nacional de Saúde Bucal. Cad. Saúde Pública, 28(1), 20-29.

Garbin, C. A., Chiba, F. Y., Garbin, A. J., \& Arcieri, R. M. (2011). Prevalência de cárie dentária em pré-escolares de escolas de educação infantil de Araçatuba. Rev Odontológica de Araçatuba, 32(2), 28-32.

Garbin, C. A., Soares, G. B., Docusse, F. R., Garbin, A. J., \& Arcieri, R. M. (2005). Oral health education in school: parents' attitudes and prevalence of caries in children. Rev Odontol UNESP, 44(5), 285-291.

Hashim, R., Williams, S., \& Thomson, W. (2009). Diet and caries experience among preschool children in Ajman, United Arab Emirates. Eur J Oral Sci, 117(1), pp. 634-740.

Jorge, A. O. (2012). Microbiologia e Imunologia Oral. Elsevier.

Junior Lima, J. L., Gonçalves, L. V., \& Correia, A. A. (2015). limentos x cárie: a ingestão do açúcar em excesso como fator estimulante do desenvolvimento da doença. ev Ciências Biológicas e da Saúde, 2(2), pp. 11-20.

Limeira, A. B., Lima, F. R., França, C., Colares, V., \& Grinfeld, S. (2010). Prevalência de cáries em crianças e cuidadores de uma creche em Recife/Pe. Rev Odontologia Clínico-Científica.

Mahan, L. A. (1995). Alimentos, nutrição e dietoterapia (8a ed.), Roca.

Moreira, P. R. (2016). Práticas alimentares relacionadas à cárie dentária: uma revisão. Universidade Federal do Rio Grande do Sul. 
Research, Society and Development, v. 10, n. 7, e52310713977, 2021

(CC BY 4.0) | ISSN 2525-3409 | DOI: http://dx.doi.org/10.33448/rsd-v10i7.13977

Nunes, V. H., \& Perosa, G. B. (2017). Cárie dentária em crianças de 5 anos: fatores sociodemográficos, lócus de controle e atitude parentais. Ciência \& Saúde Coletiva, 22(1), 191-201.

Parisotto, T., Stripp, R., Rodrigues, L., Matios-Graner, R., Costa, L., \& Nobre-dos-Santos, M. (2015). aninsoluble polysaccharide concentration in dental plaque, sugar exposure and cariogenic microorganisms predicte arly childhood caries? A follow-up study. Archives Of Oral Biology, 60(1), $1091-1097$.

Seow, K. W. (1998). Biological mechanisms of early childhood caries. Rev Community Dent Oral Epidemiol, 26(1), 8-27.

Silva, J. M. (2007). Avaliação da frequência do consumo de alimentos cariogênicos e sua relação com a cárie dentária numa amostra de crianças. Universidade do Porto, Faculdade de Ciências da Nutrição e Alimentação.

Vasconcelos, F. G., Gondim, B. L., Rodrigues, L. V., Lima Neto, E. A., \& Valença, A. M. (2018). Evolução dos índices CEO-D/CPO-D e de cuidados odontológicos em crianças e adolescentes com base no SB Brasil 2003 e SB Brasil 2010. R Bras Ci Saúde, 24(4), 333-340.

Vega, L. R., Botiel, L. B., Mazo, L. D., Torres, I. R., \& Dominguez, Y. B. (2014). Caries dental en adolescentes de una comunidade venezolana. Rev Medisan Santiago de Cuba, 18(8), 1043. 